\title{
Clinical Profile of Acute Pancreatitis in Children in a Tertiary Level Hospital of Bangladesh
}

\author{
NADIRA MUSABBIR ${ }^{1}$, ASM BAZLUL KARIM ${ }^{2}$, MD. WAHIDUZZAMAN MAZUMDER ${ }^{3}$, KANIZ SULTANA $^{4}$, \\ SYEDA AFRIAANWAR ${ }^{5}$, MD. ARIFUL HAQUE ${ }^{6}$, FAIKA HUSSAIN $^{7}$
}

\begin{abstract}
Background: Acute pancreatitis is an acute inflammatory condition of the pancreas that may extend to local and distant extrapancreatic tissues. The incidence of acute pancreatitis in children has increased significantly in the past two decades. It can be associated with severe morbidity and mortality. It should be considered in every child with unexplained acute abdominal pain.

Objectives: To observe the clinical, biochemical and imaging profiles of acute pancreatitis in children.

Methods: It was a cross-sectional study conducted at the Department of Pediatric Gastroenterology \& Nutrition of Bangabandhu Sheikh Mujib Medical University, Dhaka from January 2014 through June 2015. A total of 50 cases of acute pancreatitis were included in this study. The diagnosis of acute pancreatitis was based on diagnostic criteria of acute pancreatitis made by INSPPIRE group (If a child had any 2 of the 3 criteria: the abdominal pain compatible with acute pancreatitis, elevated serum amylase and lor lipase level more than three times of upper limit of normal, imaging findings compatible with acute pancreatitis). Clinical characteristics, laboratory and imaging profile of the cases, complications were studied.

Results: Among 50 cases, male were $46 \%$ and male female ratio was 0.8:1. Mean age at presentation was $10.2 \pm 3.2$ years. Forty eight (96\%) patients had abdominal pain which was severe agonizing in $81.3 \%$ cases. The common location of pain was in epigastric region (77\%). Pain radiating to back in $22.9 \%$ patients. Mean duration of pain was $6.6 \pm 4.4$ days before hospital admission. Vomiting was present in $72 \%$ patients followed by fever (30\%). Two (4\%) patients had jaundice. Ascites was noted in $12 \%$ patients and abdominal mass in $6 \%$ patients. Out of 50 cases of AP, biliary sludge was associated in $6 \%$ patients, biliary ascariasis in $4 \%$, choledochal cyst in $2 \%$ and gallbladder stone in $2 \%$ patients. But in this study, $4 \%$ patients had Wilson disease. Laboratory tests showed leukocytosis in $28 \%$ patients, high serum amylase and lipase level in $56 \%$ and $58 \%$ patients respectively. Postive findings in ultrasonogram were present in $66 \%$ patients. In the present study, hypocalcemia was found in $38 \%$ patients, pseudocyst in $6 \%$ and pancreatic necrosis in $2 \%$ patients.
\end{abstract}

Conclusion: Although acute pancreatitis may present with varieties of clinical feature, the most common one is abdominal pain and common location of pain is in epigastric region. For confirmation of clinically diagnosed pancreatitis, both serum amylase and lipase level and abdominal ultrasound are useful tools.

Keywrds: Clinical Profile, Acute pancreatitis.

1. MD (Paediatric Gastroenterology), Bangabandhu Sheikh Mujib Medical University, Dhaka.

2. Chairman, Department of Paediatric Gastroenterology and Nutrition, Bangabandhu Sheikh Mujib Medical University, Dhaka.

3. Assistant Professor, Department of Paediatric Gastroenterology and Nutrition, Bangabandhu Sheikh Mujib Medical University, Dhaka.

4. MD (Paediatric Gastroenterology), Bangabandhu Sheikh Mujib Medical University, Dhaka.

5. MD (Paediatric Gastroenterology), Bangabandhu Sheikh Mujib Medical University, Dhaka.

6. MD (Paediatric Gastroenterology), Bangabandhu Sheikh Mujib Medical University, Dhaka.

7. MD (Paediatric Gastroenterology), Bangabandhu Sheikh Mujib Medical University, Dhaka.

Correspondence: Dr. Nadira Musabbir, 41/23, Gha, Salek Road, Jigatola, Dhaka- 1209. E-mail: nadira_mou @yahoo.com 


\section{Introduction}

Acute pancreatitis is defined as the histological presence of inflammation of the pancreatic parenchyma. It is a reversible process characterized by the presence of interstitial oedema, infiltration by inflammatory cells and variable degrees of cellular apoptosis, necrosis and haemorrhage. ${ }^{1}$ Though acute pancreatitis is more common in adult, but its incidence in children has increased significantly over the past few decades. There are 3.6 to 13.2 paediatric cases per 100,000 individual per year, an incidence that approaches the incidence of disease in adults. ${ }^{2}$ Due to greater physician's awareness and concurrent increase in biochemical testing for pancreatitis, the diagnosis of pancreatitis is increasing. Acute pancreatitis occurs in all age groups, even in infants ${ }^{2}$. In adult, the majority of cases of AP are attributable to gallstones and alcohol. ${ }^{3}$ But the etiologies of acute pancreatitis in children are varied and quite different from those of adults. The causes of acute pancreatitis in children are biliary, drug, idiopathic, systemic disease, trauma, infection, metabolic and hereditary. ${ }^{213}$ The clinical picture of acute pancreatitis is heterogeneous in children and therefore a high level of suspicion is required even in absence of typical symptoms. Most commonly a child with acute pancreatitis presents with abdominal pain (80 to $95 \%$ ) which is commonly located in epigastric region. The second most common symptom is nausea or vomiting (40 to $80 \%$ ). Abdominal distension is seen in $21 \%$ to $46 \%$ of patients. Other symptoms are fever, jaundice, ascites and pleural effusion. A palpable abdominal mass may also be present. According to final consensus at the INSPPIRE (International Study Group of Pediatric Pancreatitis: In search for cure) meeting in December 2010 and May 2011, acute pancreatitis requires at least two of the three criteria: $u$ 1) Abdominal pain suggestive or compatible with acute pancreatitis (abdominal pain of acute onset, especially in the epigastric region), 2) Serum amylase and /or lipase activity at least 3 times greater than upper limit of normal, 3) Imaging findings compatible with acute pancreatitis. Treatment of acute pancreatitis relies on maintaining fluid and electrolyte balance, relief of pain and treatment of underlying causes. The early diagnosis of acute pancreatitis has a crucial impact on treatment policy but the early and effective diagnosis is very much challenging. On the other hand, acute pancreatitis is very much neglected disease in Bangladesh and the actual scenario of the disease in children is unknown to us. Hence, this study has been designed to evaluate the common clinical, biochemical and imaging profiles of acute pancreatitis in children in a tertiary care center of Bangladesh.

\section{Materials and Methods}

It was a cross-sectional descriptive study carried out in the Department of Pediatric Gastroenterology \& Nutrition, Bangabandhu Sheikh Mujib Medical University, Dhaka from January 2014 through June 2015. During the study period, a total 65 cases were enrolled first. Out of them, 50 cases were included in the study based on inclusion criteria. Children were included if they fulfilled any two of the following three criteria: 1)Abdominal pain suggestive of, or compatible with acute pancreatitis (abdominal pain of acute onset, especially in the epigastric region get relieved on leaning forward), 2) Serum amylase and/ or lipase level greater than at least three times of the upper limit of normal, 3) Imaging like USG, CT scan or MRCP findings characteristic of, or compatible with acute pancreatitis. Children were excluded if they had any one of the following: 1) children having chronic pancreatitis, 2) Abdominal pain due to any surgical causes, 3) unwilling to give consent.

After recruitment, clinical history, examination findings and investigation reports were recorded in a standard data sheet.

In each case, history was taken in details especially regarding abdominal pain (onset, characteristic, location, intensity, radiation and duration of pain). Associ-ated symptoms like nausea, vomiting, loss of appetite, jaundice, fever, abdominal distension were also noted. Other history like history of drug intake, trauma, mumps and family history of pancreatitis were recorded. Examination of each case was done with especial reference to vital signs, hemodynamic stability and abdominal status like tenderness, guarding, abdominal distension, epigastric fullness, presence of ascites and bowel sound. Blood for amylase, lipase, complete blood count with haematocrit, serum calcium, blood glucose, serum creatinine, fasting lipid profile, serum ALT, HBsAg, ANA, tTG (IgA) were evaluated. Imaging techniques like plain X-ray abdomen and abdominal ultrasonography were done for establishing diagnosis and complications. Enhanced CT scan of abdomen was carried out when no visualization of the pancreas was found at USG or to better define the extent of pancreatic necrosis. ERCP and MRCP were done when feasible.Prior to the commencement of the study, the objectives were explained to the parents 
and consent was taken. It was assured that all information and records would be kept confidential.

Statistical analysis was done using Statistical Package of Social Science (SPSS) version 17 (SPSS Inc, Chicago, IL, USA) for Windows XP. All data were expressed as mean \pm standard deviation (SD) or number or percent as appropriate. Pearson correlation coefficient test was used for detection of correlation. For statistical test, $p$ value $<0.05$ was considered as significant.

\section{Results}

A total of 50 patients were enrolled in the study. Age range at diagnosis was 19 months to 15 years, mean $( \pm$ SD) age being $10.2 \pm 3.2$ years. Male female ratio was 0.8:1.

Abdominal pain (48, 96\%) and vomiting (36, $72 \%)$ were the two most common clinical features, followed by fever $(15,30 \%)$ (Table I). The common location of pain was in epigastric region $(77 \%)$ with radiation to back in $22.9 \%$ patients. Pain was severe agonizing in nature in $81.3 \%$ cases (Table II).

Table I

Clinical variables of the studied patients $(n=50)$

\begin{tabular}{lcc}
\hline Clinical features & No. of patients & Percent \\
\hline Abdominal pain & 48 & 96.0 \\
Vomiting & 36 & 72.0 \\
Fever & 15 & 30.0 \\
Jaundice & 02 & 4.0 \\
Epigastric tenderness & 41 & 82.0 \\
Abdominal guarding & 21 & 42.0 \\
Ascites & 06 & 12.0 \\
Abdominal mass & 03 & 6.0 \\
\hline
\end{tabular}

Table II

Character of abdominal pain in the studied patients $(n=48)$

\begin{tabular}{lcc}
\hline Abdominal pain & No. of patients & Percent \\
\hline Location & & \\
$\quad$ Epigastric region & 37 & 77.0 \\
$\quad$ Diffuse & 07 & 14.5 \\
$\quad$ Other & 04 & 8.3 \\
Character & & \\
$\quad$ Severe agonising & 39 & 81.3 \\
$\quad$ Dull aching & 09 & 18.7 \\
Radiation to back & 11 & 22.9 \\
Relieved by forward bending & 26 & 54.1 \\
Exacerbated after taking & 23 & 47.9 \\
heavy meal & & \\
$\quad$ Duration of abdominal pain: & & \\
Mean \pm SD* (days) & $6.6 \pm 4.4$ & \\
\hline
\end{tabular}

In this study, the associated conditions with acute pancreatitis were also searched. Out of 50 cases, biliary sludge was present in $6 \%$ patients, biliary ascariasis in $4 \%$, choledochal cyst in $2 \%$ and gallbladder stone in $2 \%$ patients. But in this study, $4 \%$ patients had Wilson disease. (Fig.-1)

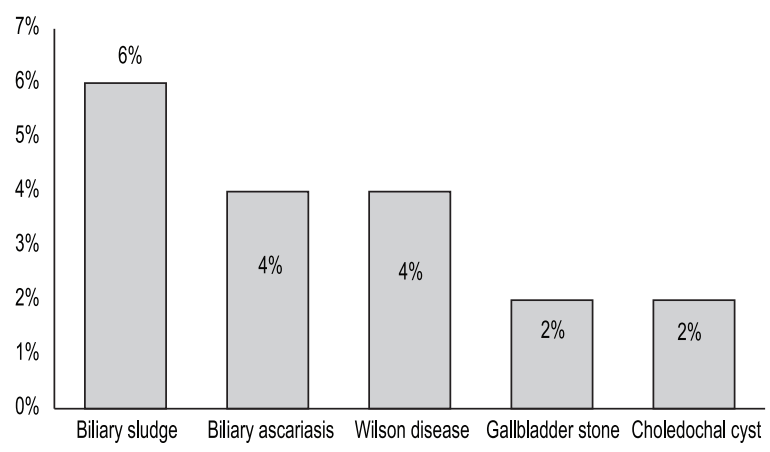

Fig.-1: Bar diagram showing distribution of associated conditions with AP $(n=50)$

Serum amylase and serum lipase value more than 3 times of upper limit of normal is considered significant for diagnosis of AP. In this study, during admission serum amylase and lipase value found to be more than 3 times of upper limit of normal in $56 \%$ patients and $58 \%$ patients consequently. In $40 \%$ cases, s. amylase and lipase level were both elevated. Median serum amylase level was $500 \mathrm{U} / \mathrm{L}$ and median serum lipase level was $1045 \mathrm{U} / \mathrm{L}$. Moderately negative correlation $(r=-0.55)$ was observed of serum amylase level with day at presentation and it was found statistically significant (Fig.-2). In case of serum lipase, moderately positive correlation $(r=0.50)$ was found from $1^{\text {st }}$ to $5^{\text {th }}$ day of presentation then from $6^{\text {th }}$ to $14^{\text {th }}$ day of presentation, strong negative correlation $(r=-0.75)$ was found and both were statistically significant (Fig.-3, 4).

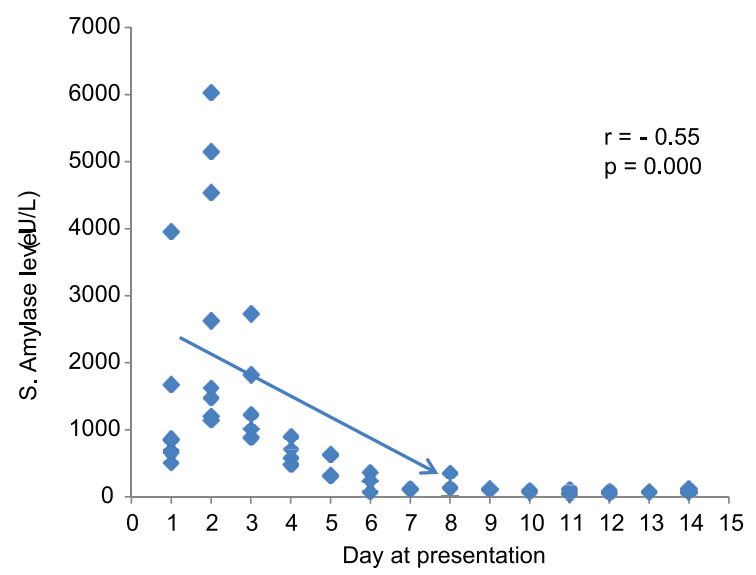

Fig.-2: Scatter diagram showing correlation of s. amylase level with day of presentation $(n=50)$ 


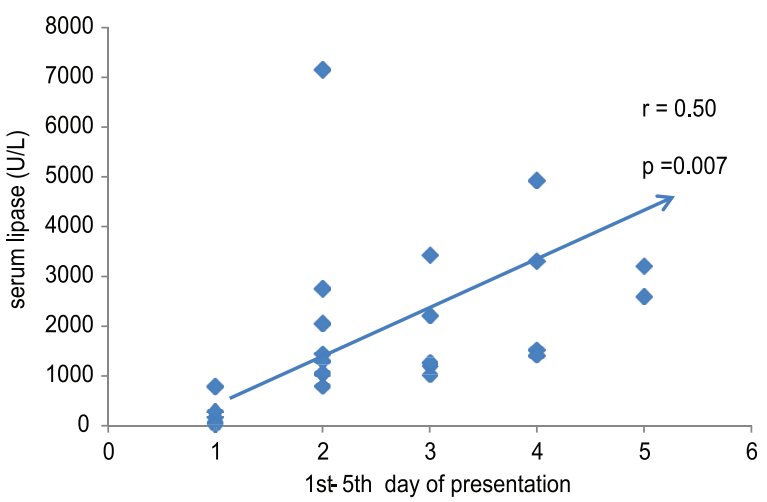

Fig.-3: Scatter diagram showing correlation of s. lipase level from $1^{\text {st }}-5^{\text {th }}$ day of presentation $(n=27)$

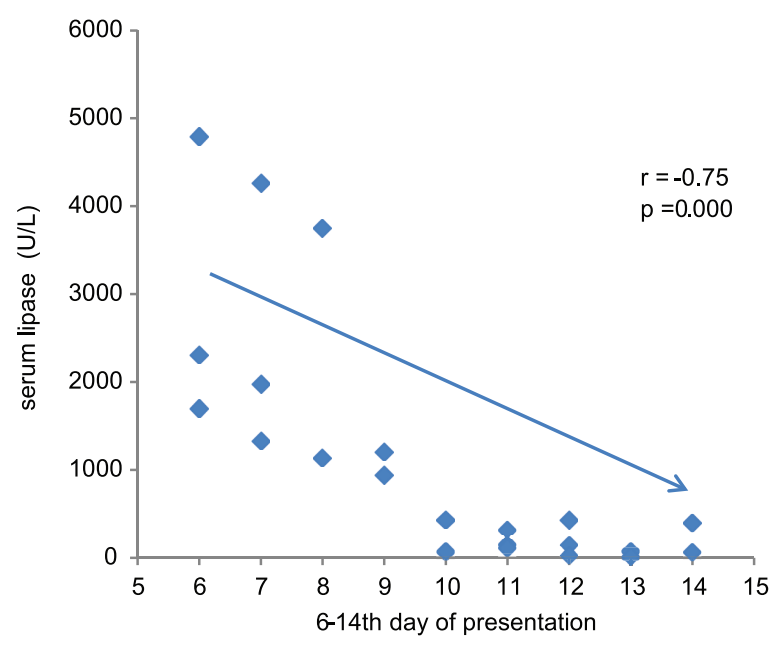

Fig.-4: Scatter diagram showing correlation of s. lipase level from $6^{\text {th }}-14^{\text {th }}$ day of presentation $(n=23)$

When other laboratory findings were analyzed, hypocalcemia was found in $38 \%$ cases, leukocytosis in $28 \%$ cases and blood glucose was within normal limit in all 50 patients.

Abdominal X-ray and USG were done in all 50 patients, among them $12 \%$ patients had abnormal $X$-ray findings (Figure: 5 ) and $66 \%$ patient had abnormal USG findings (Table III).

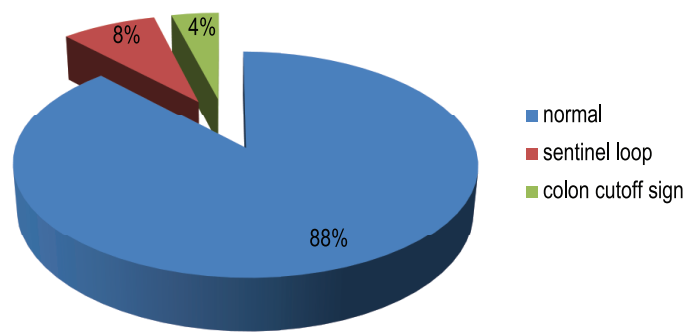

Fig.-5: Pie chart showing abdominal $x$-ray findings of the studied patients $(n=50)$
Table III

USG findings of the studied patients $(n=50)$

\begin{tabular}{lcc}
\hline USG finding & No. of patients & Percent \\
\hline Normal & 17 & 34.0 \\
Edematous or enlarged pancreas & 22 & 44.0 \\
Dilated pancreatic duct & 08 & 16.0 \\
Peripancreatic or peritoneal fluid & 06 & 12.0 \\
Dilated Common bile duct & 04 & 8.0 \\
Biliary sludge & 03 & 6.0 \\
Pseudocyst & 03 & 6.0 \\
Gallbladder Stone & 01 & 2.0 \\
\hline
\end{tabular}

Computed tomography scan was done in $28 \%$ patients and of these $78.6 \%$ patients showed abnormal findings. MRCP was done in 4 patients and all of them had abnormal findings.

Among the USG findings, 34\% patient had normal pancreas, edematous pancreas found in $44 \%$ cases, dilated pancreatic duct in $16 \%$ patient, peripancreatic fluid in $12 \%$ patient, dilated common bile duct in $8 \%$ patients, biliary sludge and pseudocyst in $6 \%$ patients and gallbladder stone in $2 \%$ patient.

Among the complications, $38 \%$ patients had hypocalcemia, peripancreatic fluid collection in $12 \%$, pseudocyst in $6 \%$ and pancreatic necrosis in $2 \%$ patients (Table IV).

Table IV

Complication of the studied patients $(n=50)$

\begin{tabular}{lcc}
\hline Complications & No of patients & Percent \\
\hline Local & & \\
Acute peripancreatic fluid & 06 & 12.0 \\
collection & & \\
Pseudocyst & 03 & 6.0 \\
Acute necrotic collection & 01 & 2.0 \\
Systemic & & \\
Hypocalcemia & 19 & 38.0 \\
\hline
\end{tabular}

\section{Discussion}

Pancreatitis is not uncommon in adults and was considered rare in pediatric population. But the incidence of acute pancreatitis is increasing day by day. Acute pancreatitis in children is a underdiagnosed disease in Bangladesh. Unfortunately no study has 
been done so far on acute pancreatitis in Bangladesh among pediatric patients. So the actual scenario of this treatable disease is not known. In this respect the picture of this disease from this study will help pediatricians to clarify some clinical aspects of this condition.

The present study was carried out with an aim to observe the clinical, biochemical and imaging profiles of acute pancreatitis in children. A total of 50 patients with acute pancreatitis who fulfilled the diagnostic criteria were included in the study.

Usually acute pancreatitis can affect all age groups, but it is more common in 5-15 year age group.v In the present study, among fifty patients, $52 \%$ were aged more than 10 years, $44 \%$ in the $5-10$ year age group and only $4 \%$ were aged less than 5 year. The mean age at presentation was $10.2 \pm 3.2$ years. The lowest age at presentation was 19 months. No case was observed in infant under the age of one year. In a large study with 279 cases of acute pancreatitis conducted by Nydegger et al.w found some different scenarios. They demonstrated that in their study $43.7 \%$ cases were in $10-15$ year age group, $31.9 \%$ cases in $5-10$ year group and $24.4 \%$ were under 5 year age group. In the present study, 54\% patients were female. Henedina et al. $x$ also found in their study that $59.5 \%$ patients were female.

The diagnosis of acute pancreatitis can be made with reasonable certainty on the basis of clinical, radiological and laboratory findings.y An overwhelming majority of patients of the present study presented with abdominal pain $(48,96 \%)$, mostly described as epigastric $(77 \%)$ followed by diffuse $(14.5 \%)$ in location. Epigastric pain radiating to the back was noted in $22.9 \%$ of patients. Mean duration of pain was $6.6 \pm 4.4$ days before hospital admission. Among the 48 patients, $81.3 \%$ had severe agonizing pain and $18.7 \%$ had dull aching pain. It was noted that only two patients did not experience abdominal pain. Vomiting was the second most frequent symptom (72\%) followed by fever (30\%). Jaundice was diagnosed in $4 \%$ cases who were subsequently found to have biliary ascariasis and a palpable mass was detected $6 \%$ of patients which was due to pseudocyst. Ascites was found in 6 patients and ascitic fluid study was done in 4 of them and showed high amylase level. Similar study was conducted by Henedina et alx , which showed abdominal pain was the most common presenting symptom in children occurring in $97.3 \%$ of cases and the most common location of pain was as follows : epigastric $(70.3 \%)$ and diffuse $(27 \%)$. In about $13.5 \%$ of cases pain radiated to back. Vomiting was present in $45.9 \%$ of cases followed by fever $(10.8 \%)$. Other symptoms such as jaundice and palpable mass were present respectively in $8.1 \%$ and $5.4 \%$ of cases.

Regarding the diagnostic biochemical evaluation, serum amylase and lipase are the most common serum assays employed for the diagnosis of acute pancreatitis in children. ${ }^{10}$ According to final consensus meeting by INSPPIRE, serum amylase and lipase activity three times greater than upper limit of normal is considered significant for diagnosis of acute pancreatitisu . In this study, serum amylase and lipase level were determined in all patients and amylase level more than 3 fold was discovered in $56 \%$ of patients. Serum lipase level was found to be elevated in $58 \%$ of patients. Median amylase level was $500 \mathrm{U} / \mathrm{L}$ (range 44-6013 U/L) and median lipase level was $1045 \mathrm{U} / \mathrm{L}$ (range 23-7148 U/L). This is comparable to findings of other studies. ${ }^{11-12}$ Park et $\mathrm{al}^{11}$ found in their study that $50 \%$ patients had serum amylase value more than 3 times upper limit of normal and $73 \%$ patients had elevated lipase value. Werlin et $\mathrm{al}^{12}$ found serum amylase $>3 x$ normal in $83 \%$ of cases and lipase $>3 x$ normal in $82 \%$ of cases. In that study, median amylase was $485 \mathrm{U} / \mathrm{L}$ and median lipase was $1841 \mathrm{U} / \mathrm{L}$. In the current study, correlation between serum amylase, serum lipase level and day at presentation of studied subjects were also observed. In acute pancreatitis, serum amylase level starts increasing from two to twelve hours after onset of symptoms and peak at 12 to 72 hours and usually returns to normal within 1 week. ${ }^{13}$ On the other hand, serum lipase level increases within 4 to 8 hours of onset of symptoms and peak at about 24 hours and level decreases within 8 to 14 days. ${ }^{13}$ In this study, it was also found that serum amylase level was elevated in patients who came on $1^{\text {st }}$ day of presentation and normal who came after $7^{\text {th }}$ day of presentation. But serum lipase level remained elevated who came within $1 \mathrm{st} 5^{\text {th }}$ day of presentation and found normal who came after $14^{\text {th }}$ day of presentation.

Regarding other laboratory investigations, it was found that haematocrit values were within normal range in all patients, total WBC count was elevated in $28 \%$ patients.

An abdominal ultrasound examination is a useful tool for diagnosis and evaluation of causes of pancreatitis. Its efficacy in diagnosing pancreatitis has been reported in $79.4 \%$ cases.x In the current study, USG was found effective in detecting pancreatitis in 66\% cases. Apart from USG which was carried out in all 
patients, imaging techniques, such as computed tomography were performed in 14 patients in whom pancreas were not visualized on USG, showing evidence of pancreatitis in $78.6 \%$ of them. Thus it is suggested that CT scan should be advised for diagnosis of pancreatitis in clinically suggestive patients when abdominal ultrasound results are not clear. Perk et al ${ }^{11}$ observed, in their retrospective study among 236 patients with acute pancreatitis, that 82 '" $6 \%$ cases underwent radiographic evaluation to establish a diagnosis or find out etiology such as common bile duct stone. Though MRCP is not routinely done in acute pancreatitis, but in this study MRCP was done in 4 patients according to opinion of hepatobiliary surgeon and all of them showed abnormal findings.

In children, only a small percentage of patients were reported to have severe complications as opposed to adults. Fewer than $6 \%$ of children developed pancreatic necrosis. ${ }^{1}$ Pseudocysts occured in $10-20 \%$ of cases. ${ }^{1} t$ In this study, only $6 \%$ patients had pseudocyst and pancreatic necrosis was observed in $2 \%$ cases. Hypocalcemia was found in $38 \%$ patients. Although transient hyperglycemia (50-70\%) is not uncommon in acute pancreatitis, permanent diabetes mellitus is exceedingly rare in children. ${ }^{1}$ In this study no patient presented with hyperglycemia.

\section{Conclusion}

Acute pancreatitis in children is an increasing health problem. Although acute pancreatitis may present with varieties of clinical feature, the most common one is abdominal pain and common location of pain is in epigastric region. For confirmation of clinically diagnosed pancreatitis, both serum amylase and lipase level and abdominal ultrasound are useful tools.

Greater awareness needs to be created about childhood acute pancreatitis and it should be considered in every children with unexplained abdominal pain so that early diagnosis of acute pancreatitis can be made and complication of the disease can be prevented by starting early treatment.

\section{References}

1. Bai HX, Lowe ME, Husain SZ. What have we learned about acute pancreatitis in children. J Pediatr Gastroenterol Nutr. 2011; 52: 262-70.

2. Srinath A, Lowe ME. Pediatric Pancreatitis. Pediatr Rev. 2013; 34: 79-89.

3. Park A, Latif SU, Shah AU, Tian J, Werlin S, Hsiao A, et al. Changing referral trends of acute pancreatitis in children: a 12 year single center analysis. J Pediatr Gastroenterol Nutr. 2009; 49: 316-22.

4. Filho EM, Carvalho WB, Silva FD. Acute pancreatitis in pediatrics: a systemic review of the literature. J Pediatr. 2012; 88: 101-14.

5. Morinville VD, Husain SZ, Bai H, Barth B, Alhosh $R$, Durie PR, et al. Definitions of pediatric pancreatitis and survey of present clinical practices. J Pediatr Gastroenterol Nutr. 2012; 55: 261-5.

6. Lautz TB, Chin AC, Radhakrishnan J. Acute pancreatitis in children: spectrum of disease and predictors of severity. J Pediatr Surg. 2011; 46: 1144-49.

7. Nydegger A, Heine RG, Ranuh R, Levy R, Crameri J, Oliver MR. Changing incidence of acute pancreati-tis: 10-year experience at the Royal Children's Hospital, Melbourne. J Gastroenterol Hepatol. 2007; 22: 1313-16.

8. Henedina A, Joao N, Aurelio M, Jorge C. Acute pancreatitis in children: a tertiary hospital report. Scand J Gastroenterol. 2014; 49: 642-647.

9. Mitchell S. Acute Pancreatitis: Etiology, clinical presentation, diagnosis, and therapy. Med Clin N Am. 2008; 92: 889-923.

10. Kandula L, Lowe ME. Etiology and outcome of acute pancreatitis in infants and toddlers. $J$ Pediatr. 2008; 152: 106-10.

11. Park AJ, Latif SU, Ahmad MU, Bultron G, Orabi $\mathrm{Al}$, Bhandari $\mathrm{V}$, et al. A comparison of presentation and management trends in acute pancreatitis between infants/toddlers and older children. J Pediatr Gastroenterol Nutr. 2010; 51:167-70.

12. Werlin SL, Kugathasan S, Frautschy BC. Pancreatitis in children. J Pediatr Gastroenterol Nutr. 2003; 37: 591-5.

13. Munoz A, Katerndahl DA. Diagnosis and management of acute pancreatitis. Am Fam Physician. 2000; 62: 164-74.

14. Ibrahim MM, Gabr K, Abdul M, Fahmi H, Booq YE. Acute pancreatitis in children: an experience with 50 cases. Ann Pediatr Surg. 2011; 7: 72-75.

15. Kota SK, Jammula S, Meher LK, Modi KD. Acute pancreatitis in association with diabetic ketoacidosis in a newly diagnosed type I Diabetes Mellitus patient; case associated review. IJCCI. 2012; 4: 54-60. 